\title{
Direct Regulation of Extracellular Chitinase Production by the Transcription Factor LeClp in Lysobacter enzymogenes OH11
}

\author{
Huiyong Xu, Hongfu Chen, Yuemao Shen, Liangcheng Du, Shan-Ho Chou, Hongxia Liu, Guoliang Qian, and Fengquan Liu \\ First, second, sixth, seventh, and eighth authors: College of Plant Protection, Nanjing Agricultural University, Nanjing 210095, P.R. China and \\ Key Laboratory of Integrated Management of Crop Diseases and Pests (Nanjing Agricultural University), Ministry of Education; third author: \\ Key Laboratory of Chemical Biology, School of Pharmaceutical Sciences, Shandong University, 250100 Jinan, P.R. China; fourth author: \\ Department of Chemistry, University of Nebraska-Lincoln, Lincoln 68588; fifth author: Institute of Biochemistry, and NCHU Agricultural \\ Biotechnology Center, National Chung Hsing University, Taichung, Taiwan, ROC; and eighth author: Institute of Plant Protection, Jiangsu \\ Academy of Agricultural Sciences, Nanjing 210014, P.R. China.
}

Accepted for publication 1 May 2016.

\begin{abstract}
Xu, H., Chen, H., Shen, Y., Du, L., Chou, S.-H., Liu, H., Qian, G., and Liu, F. 2016. Direct regulation of extracellular chitinase production by the transcription factor LeClp in Lysobacter enzymogenes OH11. Phytopathology 106:971-977.

Lysobacter enzymogenes is a gram-negative bacterial biological control agent that produces abundant extracellular enzymes capable of degrading the cell walls of fungal pathogens. In strain OH11, an isolate from China, the global regulator LeClp controls the production of extracellular chitinase by regulating the transcription of the chitinase-encoding gene chiA. Using a

combination of bioinformatic, genetic, and biochemical methods, we show that $L e \mathrm{Clp}$ regulates chiA transcription by directly binding to the chiA promoter region. Although $L e C l p$ appears to be important in this role, it is not the sole regulator of chiA transcription. Furthermore, the sequence analysis of putative LeClp binding sites indicated that the LeClp homolog could be involved in the regulation of extracellular chitinase production in diverse Lysobacter spp. by a mechanism similar to that in $L$. enzymogenes. Our findings present new insights into the molecular mechanism of $\mathrm{LeClp}$ in controlling extracellular chitinase activity, providing a fundamental road to elucidate how $L e C l p$ regulates the production of other extracellular lytic enzymes in L. enzymogenes.
\end{abstract}

Lysobacter is a gram-negative bacterial genus belonging to the family Xanthomonadaceae (Christensen and Cook 1978). This genus consists of a wide range of species from diverse ecological niches, including plant surface, rhizosphere soil, and water (Christensen and Cook 1978; Hayward et al. 2010). Considerable research attention has been paid to this genus due to its capacity to produce a multitude of new antibiotics and abundant lytic enzymes (Christensen and Cook 1978; Hayward et al. 2010; Xie et al. 2012). These characteristics led some species, such as Lysobacter enzymogenes, L. antibioticus, and L. capsici, to be important biological control agents against a variety of crop diseases (Ji et al. 2008; Ko et al. 2009; Puopolo et al. 2014; Qian et al. 2009; Zhang and Yuen 1999). Among these species, the best-studied is L. enzymogenes (Christensen and Cook 1978; Kobayashi et al. 2005; Qian et al. 2012, 2013, 2014; Zhang and Yuen 1999). As denoted by its name, this species is shown to produce extracellular lytic enzymes such as chitinases, proteases, and $\beta$-1,3-glucanases (Kobayashi et al. 2005). These enzymes have important roles in the antifungal activity of L. enzymogenes because they can efficiently degrade the cell walls of fungi (Palumbo et al. 2005; Zhang and Yuen 2000).

The role of chitinases in control of fungal crop diseases has been well demonstrated in L. enzymogenes. In an early study, treatment of conidia of the fungus Bipolaris sorokiniana with chitinolytic fractions from L. enzymogenes strain $\mathrm{C} 3$ caused conidial deformation and abnormal germ tube formation; these effects were lost when the chitinolytic fractions were heated (Zhang and Yuen 2000). In a later report,

Corresponding authors: G. Qian; E-mail address: glqian@njau.edu.cn; and F. Liu fqliu20011@sina.com

*The $\boldsymbol{e}$-Xtra logo stands for "electronic extra" and indicates that one supplementary table is published online.

http://dx.doi.org/10.1094/PHYTO-01-16-0001-R

(C) 2016 The American Phytopathological Society
L. enzymogenes $\mathrm{C} 3$ was found to produce at least two chitinases that are antifungal, including chitinase $\mathrm{A}$ and an unknown chitinase with a molecular weight of $48 \mathrm{kDa}$ (Zhang et al. 2001). These early reports demonstrated the importance of chitinase production to L. enzymogenes biocontrol activity. In this regard, understanding of the molecular mechanisms involved in the regulation of chitinase biosynthesis in L. enzymogenes will be helpful in developing methods to enhance chitinase production and, thus, improve biocontrol effectiveness.

In the first report on the genetic regulation of chitinase biosynthesis in L. enzymogenes (Kobayashi et al. 2005), chitinase production by L. enzymogenes C3 was shown to be modulated by the cAMP receptor-like protein $(\mathrm{Clp})$ transcription factor, with inactivation of clp resulting in near-complete deficiency in chitinase production. Furthermore, a recent screen of a transposon-based mutant library of strain C3 led to identification of four new regulators controlling the production of extracellular chitinase: glucose-galactose transporter, disulfide bond formation protein $\mathrm{B}$, Clp protease, and polyamine synthase (Choi et al. 2012). However, it is not clearly understood as to how these regulators function in controlling extracellular chitinase production in L. enzymogenes $\mathrm{C} 3$.

In agreement with the findings using strain $\mathrm{C} 3$, we recently showed that extracellular chitinase activity in L. enzymogenes $\mathrm{OH} 11$, a strain isolate in China, is also regulated by Clp (Qian et al. 2009; Xu et al. 2015); mutation of $c l p$ also completely abolished extracellular chitinase production in strain $\mathrm{OH} 11$. A further study showed that, although strain $\mathrm{OH} 11$ possesses three predicted chitinase-encoding genes, only chiA, whose product is chitinase A, is required for extracellular chitinase activity (Xu et al. 2015). These results suggest that Clp most likely regulates extracellular chitinase activity in L. enzymogenes $\mathrm{OH} 11$ through control of chitinase A biosynthesis or secretion. The results from investigations on strains $\mathrm{C} 3$ and $\mathrm{OH} 11$ collectively suggest that $\mathrm{Clp}$ plays a conserved role in controlling extracellular chitinase activity; however, the precise mechanism by which Clp functions in each strain remains unclear. 
The objective of the present work was to determine how Clp regulates extracellular chitinase production in L. enzymogenes, using strain $\mathrm{OH} 11$ as the subject. In the present study, the Clp of L. enzymogenes is designated "LeClp". We provide abundant evidence to show that $L e C l p$ directly binds the promoter region of chiA under both in vitro and in vivo conditions, thus resulting in a positive regulation of chiA transcription. Our studies establish the first detailed report on the mechanism by which $L e C l p$ exerts its regulation on extracellular chitinase production in L. enzymogenes. Our results also show that the direct regulatory mode of LeClp on chiA appears to be a conserved attribute in diverse chitinaseproducing Lysobacter spp.

\section{MATERIALS AND METHODS}

Bacterial strains, plasmids, and growth conditions. The bacterial strains and plasmids used in this study are listed in Table 1. Escherichia coli strains used for plasmid construction were routinely grown in Luria broth (LB) or on LB agar plates at $37^{\circ} \mathrm{C}$, supplemented with gentamicin $(\mathrm{Gm})(25 \mu \mathrm{g} / \mathrm{ml})$ or 5-bromo-4-chloro-3-indolyl$\beta$-D-galacto-pyranoside $(100 \mu \mathrm{g} / \mathrm{ml})$ as needed. L. enzymogenes stains were grown in LB medium or $1 / 10$ tryptic soy broth (TSB) at $28^{\circ} \mathrm{C}$. When required, antibiotics were added into the medium according to the following final concentrations: kanamycin $(\mathrm{Km})$ at $100 \mu \mathrm{g} / \mathrm{ml}, \mathrm{Gm}$ at $150 \mu \mathrm{g} / \mathrm{ml}$, and ampicillin (Amp) at $100 \mu \mathrm{g} / \mathrm{ml}$.

Bacterial one-hybrid assay. The bacterial one-hybrid reporter system was shown to be efficient for the testing of the physical interaction of the transcription factor with the promoter of target genes (Guo et al. 2009). This assay was applied to examine the potential interaction between the transcriptional regulator ( $L e C l p)$ and the target DNA (the chiA promoter) in the present study. As described previously, the bacterial one-hybrid reporter system involved three components: plasmids $\mathrm{pBXcmT}$ and $\mathrm{pTRG}$, which were used for cloning the bait DNA and expressing a target protein, respectively, and E. coli XL1-Blue MRF' kan strain (Table 1), which is the host strain for propagating $\mathrm{pBXcmT}$ and $\mathrm{pTRG}$ recombinants (Guo et al. 2009). In the present study, the chiA promoter region (400 bp) of L. enzymogenes OH1 1 was cloned into pBXcmT, generating the recombinant vector $\mathrm{pBXcmT}$-chiA (Table 1). Similarly, the coding region of Leclp (690 bp) was cloned into pTRG, creating the final construct pTRG-clp (Table 1). The vector pBXcmTchiA and pTRG-clp were transformed into XL1-Blue MRF' kan strain. If the direct physical binding occurs between LeClp and the chiA promoter, the positive-transformed $E$. coli strain containing both pBXcmT-chiA and pTRG-clp will be expected to grow well on the selective medium, which is the minimal medium containing $5 \mathrm{mM}$ 3-amino-1,2,4-triazole, streptomycin at $8 \mu \mathrm{g} / \mathrm{ml}$, tetracycline at $12.5 \mu \mathrm{g} / \mathrm{ml}$, chloramphenicol at $34 \mu \mathrm{g} / \mathrm{ml}$, and $\mathrm{Km}$ at $30 \mu \mathrm{g} / \mathrm{ml}$, as described previously (Guo et al. 2009). Furthermore, the cotransformant containing the vector pBX-R2031/pTRG-R3133 served as a positive control (Guo et al. 2009), while the cotransformant containing the empty pTRG and pBXcmT-chiA was used as a negative control in the present study. All cotransformants were spotted onto the selective medium and grown at $28^{\circ} \mathrm{C}$ for 3 to 4 days, then photographed.

Protein expression and purification. The assay of protein expression and purification was performed as previously described (Deng et al. 2012). In brief, the gene Leclp was amplified by polymerase chain reaction (PCR) with the designated primer pairs listed in Supplementary Table S1. The PCR product of Leclp was purified and digested by $B a m \mathrm{HI}$ and $\mathrm{XhoI}$, and further cloned into the BamHI/XhoI-digested expression vector pGEX-6p-1, creating the final construct pGEX-6p-1-clp (Table 1). This vector was transformed into E. coli strain BL21 (DE3) for protein expression. In brief, the resultant transformed strain was cultivated in LB medium containing Amp at $100 \mu \mathrm{g} / \mathrm{ml}$ overnight at $37^{\circ} \mathrm{C}$. Then, a 5-ml overnight culture was transferred into a fresh 500-ml LB medium in the presence of Amp at $100 \mu \mathrm{g} / \mathrm{ml}$ and grown at $37^{\circ} \mathrm{C}$ with shaking at $200 \mathrm{rpm}$ until the optical density at $600 \mathrm{~nm}\left(\mathrm{OD}_{600}\right)$ value reached 0.4. Subsequently, isopropyl $\beta$-D-1-thiogalactopyranoside at a final concentration of $0.4 \mathrm{mM}$ was added to the culture, followed by further growth at $28^{\circ} \mathrm{C}$ for $4 \mathrm{~h}$. Then, the cells were collected by centrifugation $(6,000 \mathrm{rpm})$ at $4{ }^{\circ} \mathrm{C}$ and resuspended in $15 \mathrm{ml}$ of phosphate-buffered saline (PBS) lysis buffer supplemented with phenylmethylsulfonyl fluoride (PMSF) at a final concentration of $1 \mathrm{mM}$. The cells were lysed by a brief sonication and the crude cell extracts were centrifuged at $13,000 \mathrm{rpm}$ and $4^{\circ} \mathrm{C}$. Soluble protein fractions were collected and mixed with preequilibrated Glutathione Sepharose 4B (GE) for $6 \mathrm{~h}$ at $4^{\circ} \mathrm{C}$, which was placed into a column and extensively washed with PBS buffer. The proteins were subsequently eluted using elution buffer that contained the

TABLE 1. Bacterial strains and plasmids used in this study

\begin{tabular}{|c|c|c|}
\hline Strains, plasmids & Characteristics $^{\mathrm{a}}$ & Source \\
\hline \multicolumn{3}{|l|}{ Lysobacter enzymogenes } \\
\hline OH11 & Wild-type strain, $\mathrm{Km}^{\mathrm{R}}$ & Qian et al. 2009 \\
\hline$\Delta$ Leclp & Leclp in-frame deletion mutant of strain $\mathrm{OH} 11, \mathrm{Km}^{\mathrm{R}}$ & Wang et al. 2014 \\
\hline$\Delta$ Leclp (pBBR) & Mutant $\Delta$ Leclp harboring plasmid $\mathrm{pBBR} 1-\mathrm{MCS} 5, \mathrm{Km}^{\mathrm{R}}, \mathrm{Gm}^{\mathrm{R}}$ & Wang et al. 2014 \\
\hline$\Delta$ chiA & chiA in-frame deletion mutant of strain $\mathrm{OH} 11, \mathrm{Km}^{\mathrm{R}}$ & Qian et al. 2012 \\
\hline$\Delta \operatorname{chiA}(\operatorname{chiA})$ & Mutant $\Delta$ chiA harboring plasmid pBBR-chiA, $\mathrm{Km}^{\mathrm{R}}, \mathrm{Gm}^{\mathrm{R}}$ & Xu et al. 2015 \\
\hline$\Delta c h i A(\mathrm{pBBR})$ & Mutant $\Delta$ chiA harboring plasmid pBBR1-MCS5, $\mathrm{Km}^{\mathrm{R}}, \mathrm{Gm}^{\mathrm{R}}$ & Xu et al. 2015 \\
\hline DH5a & $\begin{array}{l}\mathrm{F}^{-}, \varphi 80 \text { dlacZAM15, } \Delta(\text { lacZYA-argF }) U 169, \text { deoR, recAl, endA1, hsdR17 }\left(r_{k}^{-}, m_{k}^{+}\right) \text {, } \\
\text { phoA, supE44, } \lambda^{-}, \text {thi-1, gyrA96 }\end{array}$ & Lab collection \\
\hline BL21 (DE3) & $\mathrm{F}^{-}$, ompT, gal, dcm, lon, hsdSB $\left(r_{B}^{-}, m_{B}^{-}\right), \lambda(D E 3)$ & Lab collection \\
\hline XL1-Blue MRF’ Kan & $\begin{array}{l}\Delta(\text { mcrA }) 183, \Delta(\text { mcrCB-hsdSMR-mrr }) 173, \text { endA1, supE44, thi-1, recA1 gyrA96, } \\
\left.\text { relA1, lac, [F'proAB lacIqZAM15 Tn5 }\left(\mathrm{Km}^{\mathrm{R}}\right)\right]\end{array}$ & Guo et al. 2009 \\
\hline \multicolumn{3}{|c|}{ 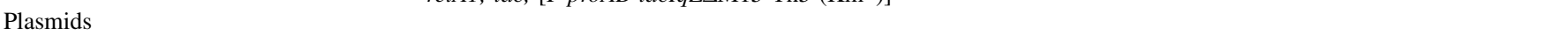 } \\
\hline pMD19-T & ColE1 origin, $\mathrm{T}$ Simple vector, $\mathrm{Amp}^{\mathrm{R}}$ & $\mathrm{TaKaRa}$ \\
\hline pTRG-clp & pTRG with the coding region of Leclp, Tet $^{\mathrm{R}}$ & This study \\
\hline $\mathrm{pBXcmT}$ & Plasmid used for DNA cloning in bacterial one-hybridization assay, $\mathrm{Chlo}^{\mathrm{R}}$ & Guo et al. 2009 \\
\hline pBXcmT-chiA & $\mathrm{pBXcmT}$ with the predicted chiA promoter region, $\mathrm{Chlo}^{\mathrm{R}}$ & This study \\
\hline
\end{tabular}

${ }^{\mathrm{a}} \mathrm{Km}^{\mathrm{R}}, \mathrm{Gm}^{\mathrm{R}} \mathrm{Amp}^{\mathrm{R}}, \mathrm{Tet}^{\mathrm{R}}$, and $\mathrm{Chlo}^{\mathrm{R}}=$ resistant to kanamycin, gentamicin, ampicillin, tetracycline, and chloramphenicol, respectively. 
reduced glutathione. Protein purity was assessed by sodium dodecyl sulfate polyacrylamide gel electrophoresis (SDS-PAGE) and protein concentration was determined using a Bradford protein assay kit (Bio-Rad).

Electrophoretic mobility shift assay. The electrophoretic mobility shift assay (EMSA) was performed as described previously (Wang et al. 2013). In brief, DNA probes (the 400-bp chiA promoter region) were amplified by PCR with the primers pchiA-F and pchiA-R. The DNA probes used in this study were labeled with $\left[\alpha-{ }^{32} \mathrm{P}\right]-\mathrm{dCTP}$ by DNA polymerase Klenow fragments, and free [ $\alpha-32 \mathrm{P}]-\mathrm{dCTP}$ was removed by ProbeQuant G-50 column (GE). The GST-tagged protein LeClp was incubated with $6 \mathrm{fmol}$ of radiolabeled oligonucleotide duplex in a $20-\mu$ reaction containing $10 \mathrm{mM}$ Tris ( $\mathrm{pH} 7.0), 50 \mathrm{mM}$ $\mathrm{KCl}, 1 \mathrm{mM}$ dithiothreitol ( $\mathrm{pH} 7.5$ ), $2.5 \%$ glycerol, $5.0 \mathrm{mM} \mathrm{MgCl}$, $1.0 \mu \mathrm{g}$ poly(dI:dC), $0.05 \% \mathrm{NP}-40$, and $10 \mathrm{mM}$ EDTA for $20 \mathrm{~min}$ at room temperature. In competition experiments, unlabeled probes were added during the preincubation period. Free DNA and protein-bound DNA were separated on $5 \%$ nondenaturing polyacrylamide gel in $0.5 \times$ Tris-boric acid-EDTA. After electrophoresis, gels were blotted onto filter paper, dried, and autoradiographed.

Chromatin immunoprecipitation PCR. The chromatin immunoprecipitation (ChIP) assay was conducted as previously described (Wang et al. 2013). In brief, L. enzymogenes stain $\mathrm{OH} 11$ was incubated in $\mathrm{LB}$ medium at $28^{\circ} \mathrm{C}$ with shaking at $200 \mathrm{rpm}$, until the value of $\mathrm{OD}_{600}$ reached 1.0. Then, the cells were harvested by centrifugation at 6,000 rpm and suspended in an equivalent volume of $10 \%$ TSB broth at $28^{\circ} \mathrm{C}$ in a rotary shaker at $200 \mathrm{rpm}$ for $1 \mathrm{~h}$. All bacterial cells were incubated with $1 \%$ formaldehyde for $10 \mathrm{~min}$ at room temperature for cross-linking and subsequently quenched with $0.5 \mathrm{M}$ glycine for $5 \mathrm{~min}$. For DNA preparation, the cells were further washed with $10 \mathrm{ml}$ of cold PBS buffer twice, then scraped into $1.2 \mathrm{ml}$ of ChIP lysis buffer (10 mM Tris-HCl, $\mathrm{pH} \mathrm{8.0;20 \%}$ sucrose; $50 \mathrm{mM} \mathrm{NaCl}$; and $10 \mathrm{mM}$ EDTA) and $4.8 \mathrm{ml}$ of ChIP buffer (50 mM HEPESKOH, pH 7.5; $150 \mathrm{mM} \mathrm{NaCl} ; 1 \mathrm{mM}$ EDTA; $1 \%$ Triton X 100; $0.1 \%$ sodium deoxycholate; $0.1 \%$ SDS; and $1 \mathrm{mM}$ PMSF). These lysates were sonicated using a Diagenode Bioruptor UCD-300 (Diagenode) to generate DNA fragments that ranged in the size from 100 to $500 \mathrm{bp}$. After centrifugation, a 100- $\mu$ l aliquot was kept as the positive control DNA (Input). The 800- $\mu$ l aliquot was incubated with $2 \mu$ of the respective antibody (anti-Clp; gift from W. Qian, Chinese Academy of Science) and $30 \mu \mathrm{l}$ of protein $\mathrm{A} / \mathrm{G}$ agarose beads overnight at $4^{\circ} \mathrm{C}$ (Sample). As a negative control, only $30 \mu \mathrm{l}$ of protein $\mathrm{A} / \mathrm{G}$ agarose beads were added in another 800- $\mu$ l aliquot (Mock). The sepharose beads were collected by centrifugation and washed with immunoprecipitation (IP) buffer, IP buffer with $500 \mathrm{mM} \mathrm{NaCl}$, wash buffer, and Tris-EDTA buffer. Then, the beads were suspended in $100 \mu$ of elution buffer $(50 \mathrm{mM}$ Tris- $\mathrm{HCl}, \mathrm{pH} 7.5 ; 10 \mathrm{mM}$ EDTA; and 1\% SDS), and incubated for $1.5 \mathrm{~h}$ at $42^{\circ} \mathrm{C}$ with RNase $\mathrm{A}$ and an additional $6 \mathrm{~h}$ at $65^{\circ} \mathrm{C}$ with proteinase $\mathrm{K}$ to reverse protein/DNA cross-links. Finally, these samples were processed for DNA purification by phenol-chloroform extraction and ethanol precipitation. The quantity of DNA was detected by the NanoDrop ND-1000 (NanoDrop). Finally, the PCR assay was performed in $25 \mu \mathrm{l}$ with the input DNA, sample DNA, and mock DNA, respectively, with the relevant primer pairs. The PCR products were separated on a $1.5 \%$ agarose gel and stained with ethidium bromide. For further validation, these PCR products were also sequenced in the present study.

Western blot analysis. The Western blot analysis was performed according to the laboratory protocol, with a minor modification $(\mathrm{Xu}$ et al. 2015). In brief, GST-tagged protein $L e C l p$ was separated by SDS-PAGE and immobilized onto a polyvinylidenem difluoride membrane using the semidry blot machine (Bio-Rad). Membranes were probed with the specific primary anti-GST (M20007; Abmart), followed by an HRP-conjugated antimouse secondary antibody (M21001; Abmart) for signal detection.

Determination of extracellular chitinase activity. The production of extracellular chitinase from L. enzymogenes strains was determined as described previously (Qian et al. 2009; Xu et al. 2015). In brief, the test L. enzymogenes strains were incubated in LB medium at $28^{\circ} \mathrm{C}$ with shaking at $200 \mathrm{rpm}$, until the value of $\mathrm{OD}_{600}$ reached 1.0. Then, $2 \mu$ of bacterial culture was spotted on LB plates contained colloidal chitin. After $96 \mathrm{~h}$ of incubation at $28^{\circ} \mathrm{C}$, chitinase activity was assessed according to the cleaning zones around the bacterial colonies. Each treatment involved three replications, and the same experiment was performed three times.

\section{RESULTS}

LeClp bound directly to the promoter region of $\boldsymbol{c h i A}$ in vitro. To explore how $L e C l p$ regulates the transcription of $c h i A$, we tested whether LeClp can directly bind the promoter of chiA via the C-terminal helix-turn-helix (HTH)-DNA binding domain of LeClp. To test this point, the characterized binding consensus sequence (5'-NTGTGA-N6-TCACANTTTN-3') of E. coli CRP (http://regulondb.ccg.unam.mx/), the homolog of $L e C l p$, was chosen for predicting whether the promoter of chiA possessed any potential DNA binding site of $L e C l p$. This led to identification of a potential binding site $\left(5^{\prime}\right.$-TTGTCACGCGGCGCCAGTTTC- $\left.3^{\prime}\right)$ of LeClp at the chiA promoter (Fig. 1A). This result supported our initial idea and further suggested that LeClp could directly bind to the $c h i A$ promoter. To validate this point, we employed the E. colibased one-hybrid reporter system (described in detail in Materials and Methods). We clearly observed that the test E. coli strain containing both $L e \mathrm{Clp}$ and the chiA promoter grew very well on the selective medium, similar to the positive control, whereas the negative control failed to grow (Fig. 1B). This result indicated that a direct binding of LeClp on the promoter of chiA occurred under the test conditions.

To further confirm the above finding, an EMSA/gel shift assay was performed. We found that the GST-tagged $\mathrm{LeClp}$ formed a clear protein/DNA complex with the test DNA probe, whereas no shift band was observed between the empty GST and DNA probe under the similar test conditions (Fig. 2). Furthermore, addition of an unlabeled probe (cold probe) at 10- and 1,000-fold excesses to the reaction mixtures competitively inhibited the binding of LeClp to the labeled DNA probe. This EMSA result, together with the data from Figure 1, strongly showed that $L e C l p$ directly bound to the promoter region of chiA under the in vitro condition.

A direct in vivo physical interaction occurred between LeClp and the chiA promoter. Next, we attempted to test whether an in vivo binding of LeClp on the promoter of chiA occurs in L. enzymogenes cells. To achieve this point, the ChIPPCR assay was carried out, whose scheme is presented in Figure 3A (described in detail in Material and Methods). Clearly, a 212-bp promoter region of chiA was amplified by PCR from the designated Sample (the chromatin DNA of strain OH11 immunoprecipitated with the respective antibody), which was similar to the case from the positive control Input (Fig. 3B). However, under the same condition, the negative control Mock (the chromatin DNA of strain OH11 immunoprecipitated without the respective antibody) failed to amplify the corresponding PCR products (Fig. 3B). These results suggested that L. enzymogenes LeClp bound to the promoter region of chiA in vivo. Collectively, all of the above results as well as our previous report (Wang et al. 2014) clearly reveal that $L e C l p$ exerted its positive regulation on $c h i A$ transcription through a direct interaction with the chiA promoter region in L. enzymogenes.

LeClp is an important but not the sole regulator of $\boldsymbol{c h i A}$ transcription. To address whether $L e C l p$ is the sole regulator of chiA transcription, the plasmid-borne chiA under the control of its native promoter was introduced into the Leclp mutant. This introduction caused a minor but visible effect in restoring the deficiency of the Leclp mutant in producing extracellular chitinase (Fig. 4). However, the Leclp mutant possessing an empty vector was still fully deficient in this ability. Furthermore, as controls, the Leclp 
or chiA mutant completely lost the ability to produce extracellular chitinase, where the trait was restored in the respective complemented strain to the wild-type level (Fig. 4). Taken together, these results suggested that, although $L e C l p$ was important, it was not the sole regulator of chiA transcription in L. enzymogenes.
The direct interaction between $L e C l p$ and the chiA promoter appears to be a conserved mechanism in diverse chitinase-producing Lysobacter spp. Recently, the genomes of four representative chitinase-producing Lysobacter spp., including L. enzymogenes C3, L. antibioticus ATCC29479, L. capsici 55, and

\section{A NTGTGANNNNNNTCACANTTTN Binding site of E.coli Crp
TTGTCACGCGGCGC CAGTTTC L.enzymogenes chiA promoter}

B

-3AT-Str

pTRG/pchiA BOH-ck(+) pTClp/pchiA

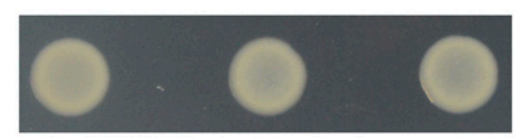

$+3 \mathrm{AT}+\mathrm{Str}^{\mathrm{r}}$

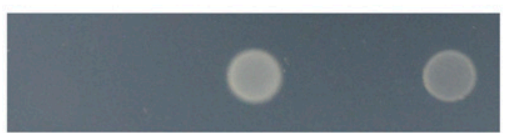

Fig. 1. LeClp directly bound to the promoter of chiA in the Escherichia coli-based one-hybrid assay. A, Alignment of the binding consensus sequence of E. coli CRP with the promoter region of chiA in Lysobacter enzymogenes. Conserved bases are highlighted in black. B, Direct physical interaction between LeClp and the chiA promoter region was detected in E. coli. Experiments were performed according to the procedures described in the Materials and Methods section. BOH$\mathrm{CK}(+)=$ cotransformant containing pBX-R2031 and pTRG-R3133, used as a positive control; pTRG/pchiA = cotranformant containing pBXcmT-chiA and the empty pTRG, served as a negative control; and pTClp/pchiA = cotranformant possessing pTRG-clp and pBXcmT-chiA (Table 1). Rows: -3 AT-Str ${ }^{\mathrm{r}}=$ no selective medium plate $(3 \mathrm{AT}=3$-amino-1,2,4-triazole and $\mathrm{Str}=$ streptomycin $)$ and $+3 \mathrm{AT}+\mathrm{Str}^{\mathrm{r}}=$ selective medium plate.
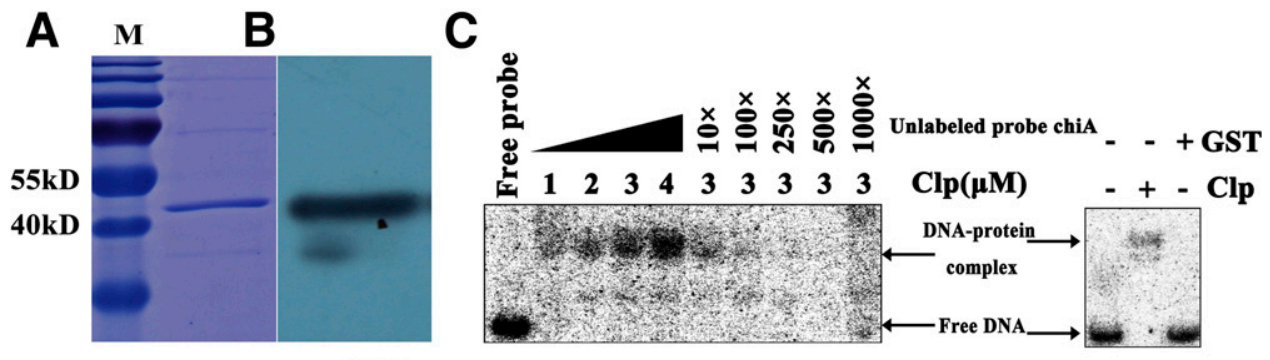

a-GST

Fig. 2. Electrophoretic mobility shift assay showing the direct binding of $L e C l p$ to the promoter of $c h i A$ in vitro. A, Sodium dodecyl sulfate polyacrylamide gel electrophoresis analysis of the purified GST-tagged $L e C l p$, which was validated by B, Western blot using $\alpha$-GST, the anti-GST antibody. C, The unlabeled probe (cold probe) at 10- and 1,000-fold excesses to the reaction mixtures competitively inhibited the binding of LeClp to the labeled DNA probe. GST was used as a negative control. Free DNA and DNA-protein complex are indicated by arrows, respectively.
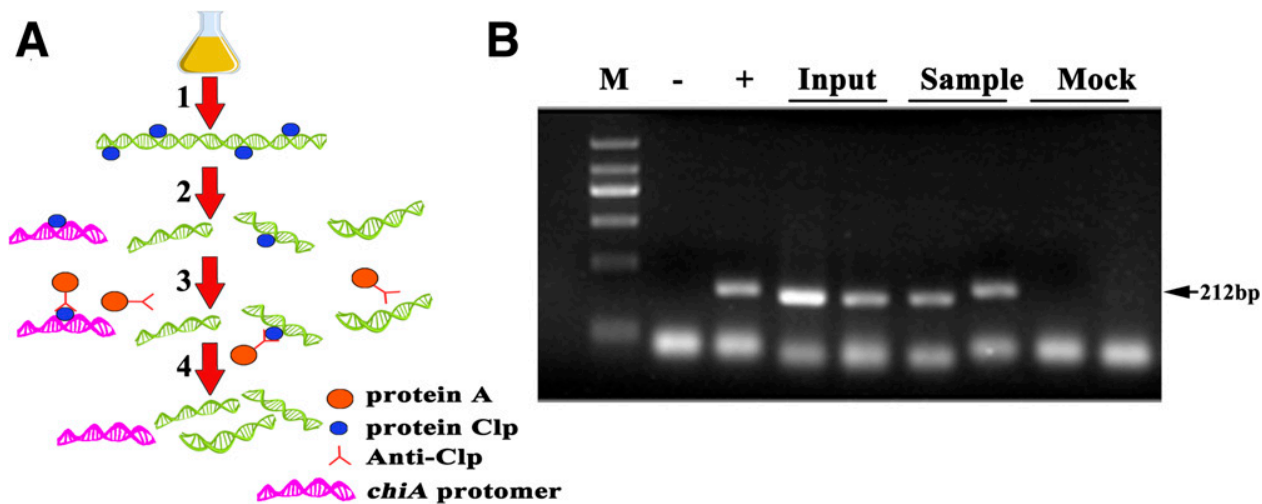

Fig. 3. LeClp directly bound the promoter of chiA in Lysobacter enzymogenes cells. A, Scheme of chromatin immunoprecipitation used in this study, including 1 , formaldehyde cross-linking; 2, sonication; 3, immunoprecipitation; and 4, reverse-crosslink. B, Results of chromatin immunoprecipitation polymerase chain reaction assay. A specific 212-bp band, belonging to the chiA promoter region, was amplified from the chromatin immunoprecipitated sample and validated by sequencing. Input = the DNA fragments that ranged in the size from 100 to $500 \mathrm{bp}$ after sonication (positive control), Sample = the chromatin DNA immunoprecipitated with the anti-cAMP receptor-like protein (Clp) antibody, Mock = the chromatin DNA immunoprecipitated without the anti-Clp antibody (negative control), $-=$ double-distilled $\mathrm{H}_{2} \mathrm{O}$ (negative control), and $+=$ the intact DNA of the wild-type strain $\mathrm{OH} 11$ (positive control). 
OH11

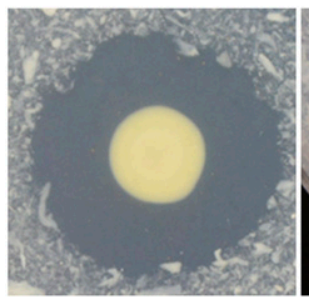

$\Delta \operatorname{Leclp}(\operatorname{chiA})$

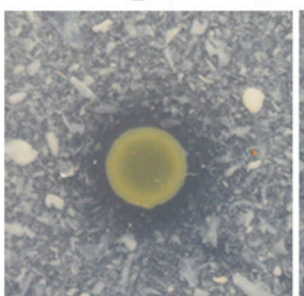

$\Delta$ Leclp

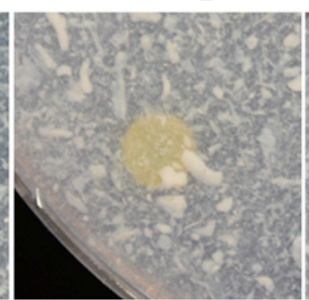

$\Delta c h i A$

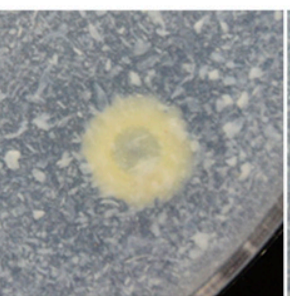

$\Delta \operatorname{Leclp}($ Leclp $)$

$\Delta \operatorname{Leclp}(\mathrm{pBBR})$

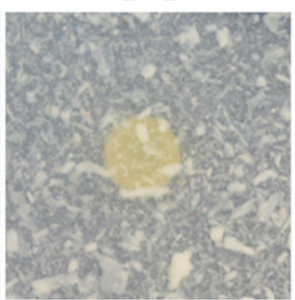

$\Delta \operatorname{chiA}(\operatorname{chiA})$

Fig. 4. LeClp was an important but not the sole regulator of chiA transcription. Production of extracellular chitinase was reflected by the hydrolyzing zone of various Lysobacter enzymogenes strains on the selective medium. $\mathrm{OH} 11$, the wild-type strain of L. enzymogenes; $\Delta$ Leclp, the clp-deletion mutant of $L$. enzymogenes; $\Delta$ Leclp(Leclp), the complemented strain of $\Delta$ Leclp; $\Delta$ Leclp(pBBR), $\Delta$ Leclp with an empty vector; $\Delta$ Leclp (chiA), $\Delta$ Leclp containing an overexpressed chiA; $\Delta$ chiA, the chiA-deletion mutant of L. enzymogenes; $\Delta$ chiA(chiA), the complemented strain of $\Delta$ chiA; and $\Delta$ chiA(pBBR), $\Delta$ chiA possessing an empty vector. Diameter of the hydrolyzing zone was indicated by a bar corresponding to $1 \mathrm{~cm}$.

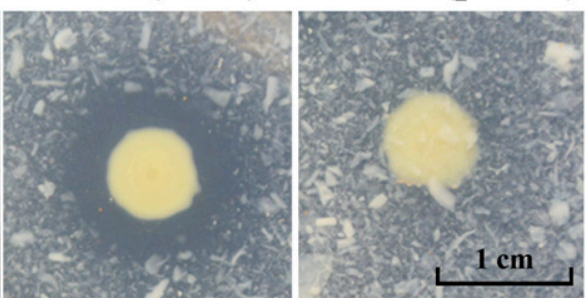

A

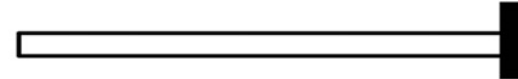

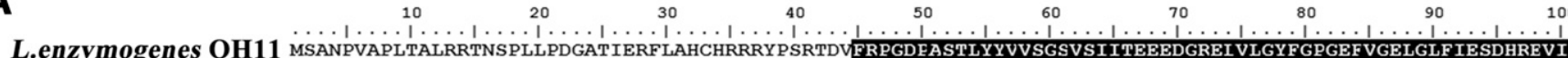

L.enzymogenes OH11 MSANPVAPLTALRRTNSPLLPDGATIERFLAHCHRRRYPSRTDVFRPGDFASTLYYVVSGSVSIITEEEDGREIVLGYFGPGEFVGELGLFIESDHREVI

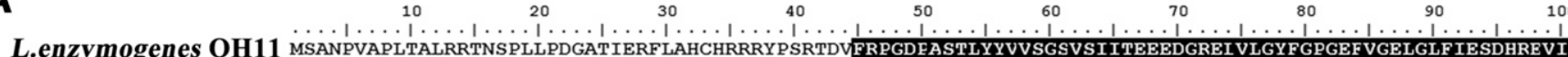
cNMP-binding L.enzymogenes $\mathrm{C} 3$ L.antibioticus ATCC29479 L.gummosus 3.2.11

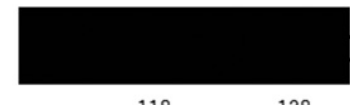

110

120

130

140 MFRPGDFASTLYYVVSGSVSI ITEEEDGREI VLGYFGPGEFVGELGLFIESDHREVI

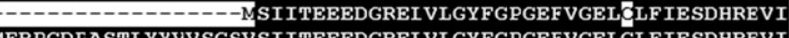
MFRPGDFASTLYYVVSGSVSIITEEEDGREIVLGYFGPGEFVGELGLF IESDHREVI

\section{HTH-Crp}

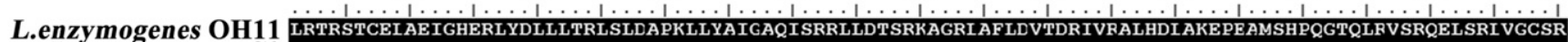
L.enzymogenes C3 LRTRSTCEI AEIGHERLYDLLLTRLSLDAPKLLYAIGAQISRRLLDTSRKAGRI AFLDVTDRIVRALHDI AKEPEAMSHPOGTQLRVSRQELSRI VGCSR L.antibioticus ATCC29479 LRTRSTCEIAEIGHERLYDLLLTRLSLDAPKLLYAIGAQISRRLLDTSRKAGRIAFLDVTDRIVRALHDIAKEPEAMSHPQGTQLRVSRQELSRIVGCSR L.capsici 55 LRTR TCEI AEIGHERLYDLLLTRLSLDA PKLLYAIGAQISRRLLDTSRKAGRI AFLDVTDRIVRALHDI AKEPEAMSHPQGTQLRVSRQELSRIVGCSR

L.gummosus 3.2.11 LRTR TCEIAEIGHERLYDLLLTRLSLDAPKLLYAIGAQISRRLLDTSRKAGRIAFLDVTDRIVRALHDI AKEPEAMSHPQGTQLRVSRQELSRIVGCSR

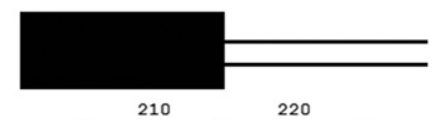

210

L.enzymogenes OH11 ENAGRVLKKLÇADGKLHARGKTVVLYGTR

L.enzymogenes C3 ENAGRVLKKLÇADGKLHARGKTVVLYGTR

L.antibioticus ATCC29479 ENAGRVLKKLÇADGKLHARGKTVVLYGTR

L.capsici 55 ENAGRVLKKLCADGKLHARGKTVVLYGTR

L.gummoSus 3.2.11 BNAGRVIKKILADGKLHARGKTVVIYGTR

B

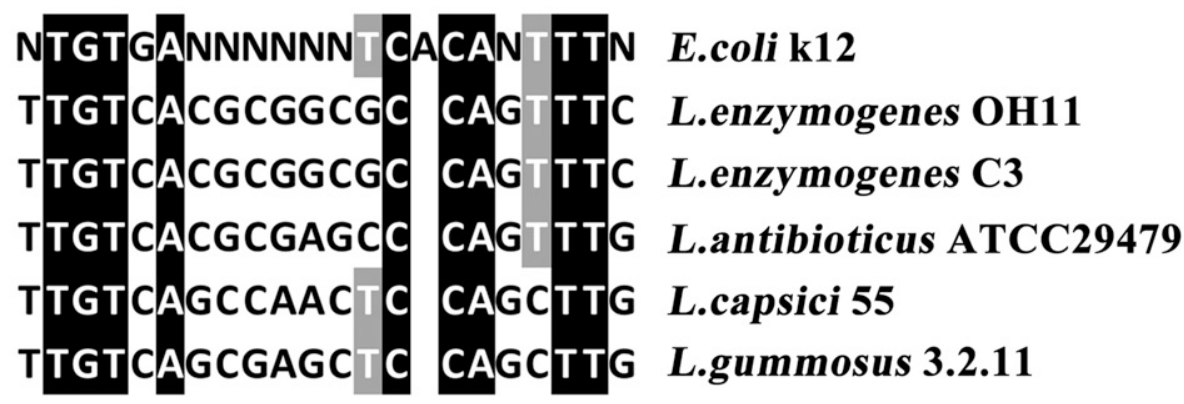

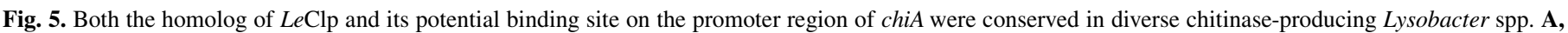

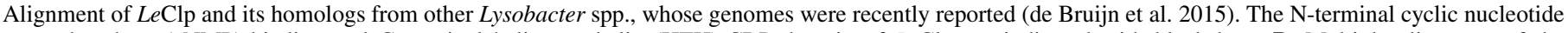

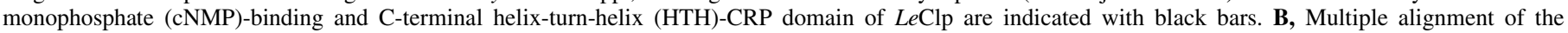

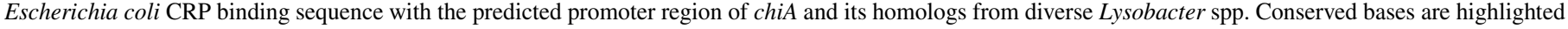
in black and gray. 
L. gummosus 3.2.11, were reported (de Bruijn et al. 2015). To explore whether the direct interaction between LeClp and the chiA promoter in strain $\mathrm{OH} 11$ is also conserved in those abovementioned Lysobacter spp., a comparative sequence alignment was performed. Clearly, all of the sequenced Lysobacter spp. possessed a highly conserved LeClp homolog (Fig. 5), and their corresponding HTHbinding domain at the amino-acid sequence level was almost same as that in strain OH11 (Fig. 5A). Furthermore, the promoter regions of chiA from all Lysobacter spp. were predicted to have a DNA binding consensus sequence of $E$. coli CRP (Fig. 5B), which was similar to the case from strain $\mathrm{OH} 11$. Collectively, the sequence analysis of the putative $L e \mathrm{Clp}$ binding site indicated that the LeClp homolog could be involved in the regulation of extracellular chitinase production in those closely related Lysobacter spp. described above, by a mechanism similar to that in L. enzymogenes OH11.

\section{DISCUSSION}

As a global regulator, $L e C l p$ makes an important contribution to the production of diverse extracellular lytic enzymes in L. enzymogenes (Kobayashi et al. 2005; Wang et al. 2014); however, the corresponding molecular mechanism is unknown. In the present study, we provided the first report to show that the regulation of $L e C l p$ on the production of extracellular chitinase was, at least in part, due to the direct binding of LeClp to the promoter of $c h i A$. Such a regulatory mechanism seems to be conserved in diverse chitinaseproducing Lysobacter spp. This new knowledge will be helpful to generate high chitinase-producing L. enzymogenes strains for better application in the biocontrol of crop fungal disease.

Although this study provides abundant evidence of the direct interaction between $L e \mathrm{Clp}$ and the chiA promoter, LeClp appears not to be the sole regulator of chiA transcription (Fig. 4). It is possible that, in addition to $L e \mathrm{Clp}$, there is another unidentified transcription factor that can bind with the native chiA promoter, causing some leaky transcription of chiA from the introduced chiA plasmids (maybe hundreds). However, in the chromosomal background of the Leclp mutant, there is only one copy of chiA; hence, the phenotype rescue phenomenon is less obvious.

The regulation of Clp on enzyme biosynthesis has been well characterized in the phytopathogenic bacterium Xanthomonas campestris pv. campestris (He et al. 2007). In this bacterium, the regulator Clp serves as a downstream regulator of the diffusible signal factor (DSF) cell-to-cell signaling to modulate diverse enzyme production (He et al. 2007). Although L. enzymogenes also contains a functional LeDSF system, this system did not regulate the biosynthesis of extracellular lytic enzymes such as chitinase (Qian et al. 2013). Therefore, in contrast to the findings in $X$. campestris, LeClp appears to utilize an LeDSFindependent manner to regulate the production of extracellular chitinase. These results suggested that the Clp family proteins have evolved to acquire species-adaptive mechanisms to control respective enzyme biosynthesis in X. campestris and L. enzymogenes, two related but different bacterial species at taxonomy.

In $X$. campestris, the regulation of Clp on extracellular enzyme biosynthesis is dependent on c-di-GMP, a newly identified bacterial second messenger (Chin et al. 2010; Tao et al. 2010). A representative example is the endoglucanase enzyme encoding by engXCA that contributes to $X$. campestris virulence through its cellulolytic activity (Gough et al. 1990). In X. campestris cells, Clp is a c-di-GMP receptor with high affinity (Chin et al.2010; Tao et al. 2010). Clp directly binds the promoter of engXCA, resulting in a positive control of its transcription. High-level c-di-GMP inhibits such binding, thus suggesting that c-di-GMP makes a negative contribution to formation of Clp-DNA complex (Chin et al. 2010; Tao et al. 2010). Although LeClp shares a high amino-acid similarity with the Clp of $X$. campestris (Kobayashi et al. 2005), it remains unclear whether the direct regulation of LeClp in transcription of chiA is c-di-GMP dependent, which needs further investigations.
In summary, our findings present new insights into how the global regulator LeClp controls the production of extracellular chitinase, which provides useful guidance to understand the regulation of $\mathrm{LeClp}$ in control of other extracellular lytic enzymes, such as $\beta-1,3-$ glucanase in L. enzymogenes. Also, our studies may trigger the study of other chitinase-producing and underexplored Lysobacter spp. possessing the homologs of both LeClp and ChiA.

\section{ACKNOWLEDGMENTS}

G. Qian is a visiting scholar in M. Gomelsky's laboratory at the University of Wyoming, supported by the China Scholarship Council. We thank W. Qian, from the Institute of Microbiology of Chinese Academy of Sciences, for providing the anti-Clp antibody as a gift, as well as his technical support in the EMSA;Z.-G. He, from Huazhong Agricultural University (China), for kindly providing the bacterial one-hybrid system; and G. Y. Yuen (University of Nebraska-Lincoln) for his critical editing. This study was supported by the National Basic Research (973) program of China (2015CB150600), National Natural Science Foundation of China (31371981, 31572046), Special Fund for Agro-Scientific Research in the Public Interest (No. 201303015) grants from Jiangsu Agriculture Science and Jiangsu Province (ZX(15)1006, BE2014386, BE2015354), Program for New Century Excellent Talents in University of Ministry of Education of China (NCET-13-0863), and Fundamental Research Funds for the Central Universities (KYTZ201403). Research in the Du lab is supported, in part, by NIH R01AI097260 and a UNL Redox Biology Center pilot grant. S.-H. Chou is supported by the Ministry of Education, Taiwan, under the ATU plan, and by the National Science Council, Taiwan (grants 102-2113-M-005-006-MY3).

\section{LITERATURE CITED}

Chin, K. H., Lee, Y. C., Tu, Z. L., Chen, C. H., Tseng, Y. H., Yang, J. M., Ryan, R. P., McCarthy, Y., Dow, J. M., Wang, A. H., and Chou, S. H. 2010. The cAMP receptor-like protein CLP is a novel c-di-GMP receptor linking cell-cell signaling to virulence gene expression in Xanthomonas campestris. J. Mol. Biol. 396:646-662.

Choi, H., Kim, H. J., Lee, J. H., Kim, J. S., Park, S. K., Kim, I. S., and Kim, Y. C. 2012. Insight into genes involved in the production of extracellular chitinase in a biocontrol bacterium Lysobacter enzymogenes C-3. Plant Pathol. J. 28:439-445.

Christensen, P., and Cook, F. D. 1978. Lysobacter, a new genus of nonfruiting, gilding bacteria with a high base ratio. Int. J. Syst. Bacteriol. 28:367-393.

de Bruijn, I., Chen, X., de Jager, V., Exposito, R. G., Watrous, J., Patel, N., Postma, J., Dorrestein, P. C., Kobayashi, D., and Raaijmakers, J. M. 2015. Comparative genomics and metabolite profiling of the genus Lysobacter. BMC Genomics 16:991.

Deng, Y. Y., Schmid, N., Wang, C., Wang, J. H., Pessi, G., Wu, D. H., Lee, J., Aguilar, C., Ahrens, C. H., Chang, C. Q., Song, H. W., Eberl, L., and Zhang, L. H. 2012. Cis-2-dodecenoic acid receptor RpfR links quorum-sensing signal perception with regulation of virulence through cyclic dimeric guanosine monophosphate turnover. Proc. Natl. Acad. Sci. USA 109: $15479-15484$

Gough, C. L., Dow, J. M., Keen, J., Henrissat, B., and Daniels, M. J. 1990. Nucleotide sequence of the engXCA gene encoding the major endoglucanase of Xanthomonas campestris pv. campestris. Gene 89:53-59.

Guo, M. M., Feng, H., Zhang, J., Wang, W. Q., Wang, Y., Li, Y. Q., Gao, C. H., Chen, H. C., Feng, Y., and He, Z. G. 2009. Dissecting transcription regulatory pathways through a new bacterial one-hybrid reporter system. Genome Res. 19:1301-1308.

Hayward, A. C., Fegan, N., Fegan, M., and Stirling, G. R. 2010. Stenotrophomonas and Lysobacter: Ubiquitous plant-associated gammaproteobacteria of developing significance in applied microbiology. J. Appl. Microbiol. 108:756-770.

He, Y. W., Ng, Y. J., Xu, M., Lin, K., Wang, L. H., Dong, Y. H., and Zhang, L. H. 2007. Xanthomonas campestris cell-cell communication involves a putative nucleotide receptor protein $\mathrm{Clp}$ and a hierarchical signaling network. Mol. Microbiol. 64:281-292.

Ji, G. H., Wei, L. F., He, Y. Q., Wu, Y. P., and Bai, X. H. 2008. Biological control of rice bacterial blight by Lysobacter antibioticus strain 13-1. Biol. Control 45:288-296.

Ko, H. S., Jin, R. D., Krishnan, H. B., Lee, S. B., and Kim, K. Y. 2009. Biocontrol ability of Lysobacter antibioticus HS124 against Phytophthora blight is mediated by the production of 4-hydroxyphenylacetic acid and several lytic enzymes. Curr. Microbiol. 59:608-615.

Kobayashi, D. Y., Reedy, R. M., Palumbo, J. D., Zhou, J. M., and Yuen, G. Y. 2005. A $c l p$ gene homologue belonging to the $c r p$ gene family globally 
regulates lytic enzyme production, antimicrobial activity, and biological control activity expressed by Lysobacter enzymogenes strain C3. Appl. Environ. Microbiol. 71:261-269.

Kovach, M. E., Elzer, P. H., Hill, D. S., Robertson, G. T., Farris, M. A., Roop, R. M., and Peterson, K. M. 1995. Four new derivatives of the broad-hostrange cloning vector $\mathrm{pBBR} 1 \mathrm{MCS}$, carrying different antibiotic-resistance cassettes. Gene 166:175-176.

Palumbo, J. D., Yuen, G. Y., Jochum, C. C., Tatum, K., and Kobayashi, D. Y. 2005. Mutagenesis of beta-1,3-Glucanase genes in Lysobacter enzymogenes strain C3 results in reduced biological control activity Toward Bipolaris leaf spot of tall fescue and Pythium damping-off of sugar beet. Phytopathology 95:701-707.

Puopolo, G., Cimmno, A., Palmieri, M. C., Giovannini, O., Evidente, A., and Pertot, I. 2014. Lysobacter capsici AZ78 produces cyclo (L-Pro-L-Tyr), a 2, 5-diketopiperazine with toxic activity against sporangia of Phytophthora infestans and Plasmopara viticola. J. Appl. Microbiol. 117: 1168-1180.

Qian, G. L., Hu, B. S., Jiang, Y. H., Fan, J. Q., and Liu, F. Q. 2009. Identification and characterization of Lysobacter enzymogenes as a biological control agent against some fungal pathogens. Agric. Sci. China 8:68-75.

Qian, G. L., Wang, Y. L., Liu, Y. R., Xu, F. F., He, Y.-W., Du, L. C., Venturi, V., Fan, J. Q., Hu, B. S., and Liu, F. Q. 2013. Lysobacter enzymogenes uses two distinct cell-cell signaling systems for differential regulation of secondary-metabolite biosynthesis and colony morphology. Appl. Environ. Microbiol. 79:6604-6616.

Qian, G. L., Wang, Y. S., Qian, D. Y., Fan, J. Q., Hu, B. S., and Liu, F. Q. 2012. Selection of available suicide vectors for gene mutagenesis using chiA (a chitinase encoding gene) as a new reporter and primary functional analysis of chiA in Lysobacter enzymogenes strain OH11. World J. Microb. Biot. 28:549-557.
Qian, G. L., Xu, F. F., Venturi, V., Du, L. C., and Liu, F. Q. 2014. Roles of a solo LuxR in the biological control agent Lysobacter enzymogenes strain OH11. Phytopathology 104:224-231.

Tao, F., He, Y. W., Wu, D. H., Swarup, S., and Zhang, L. H. 2010. The cyclic nucleotide monophosphate domain of Xanthomonas campestris global regulator Clp defines a new class of cyclic di-GMP effectors. J. Bacteriol. 192:1020-1029

Wang, F. F., Deng, C. Y., Cai, Z., Wang, T., Wang, L., Wang, X. Z., Chen, X. Y., Fang, R. X., and Qian, W. 2013. A three-component signalling system fine-tunes expression kinetics of HPPK responsible for folate synthesis by positive feedback loop during stress response of Xanthomonas campestris. Environ. Microbiol. 16:2126-2144.

Wang, Y. S., Zhao, Y. X., Zhang, J., Zhao, Y. Y., Shen, Y., Su, Z. H., Xu, G. G., Du, L. C., Huffman, J. M., Venturi, V., Qian, G. L., and Liu, F. Q. 2014. Transcriptomic analysis reveals new regulatory roles of Clp signaling in secondary-metabolite biosynthesis and surface motility in Lysobacter enzymogenes OH11. Appl. Microbiol. Biotechnol. 98:9009-9020.

Xie, Y., Wright, S., Shen, Y. M., and Du, L. C. 2012. Bioactive natural products from Lysobacter. Nat. Prod. Rep. 29:1277-1287.

Xu, G. G., Zhao, Y. X., Du, L. C., Qian, G. L., and Liu, F. Q. 2015. Hfq regulates antibacterial antibiotic biosynthesis and extracellular lytic-enzyme production in Lysobacter enzymogenes OH11. Microb. Biotechnol. 8:499-509.

Zhang, Z., and Yuen, G. Y. 1999. Biological control of Bipolaris sorokiniana on tall fescue by Stenotrophomonas maltophilia strain C3. Phytopathology 89:817-822.

Zhang, Z. G., and Yuen, G. Y. 2000. The role of chitinase production by Stenotrophomonas maltophilia strain C3 in biological control of Bipolaris sorokiniana. Phytopathology 90:384-389.

Zhang, Z., Yuen, G. Y., Sarath, G., and Penheiter, A. R. 2001. Chitinases from the plant disease biocontrol agent, Stenotrophomonas maltophilia C3. Phytopathology 91:204-211. 